Cahiers
de la Recherche
sur les Droits

Cahiers de la recherche sur les droits

Fondamentaux fondamentaux

$14 \mid 2016$

Urbanisme et droits fondamentaux

\title{
Le contentieux de l'excès de pouvoir des autorisations d'urbanisme et le droit au recours
}

Litigation for Misuse of Power in the Granting of Planning Permission and the Right of Appeal

Jean-Christophe Le Coustumer

\section{OpenEdition}

Journals

Édition électronique

URL : https://journals.openedition.org/crdf/586

DOI : $10.4000 /$ crdf.586

ISSN : 2264-1246

Éditeur

Presses universitaires de Caen

Édition imprimée

Date de publication : 1 novembre 2016

Pagination : 41-50

ISBN : 978-2-84133-838-2

ISSN : $1634-8842$

\section{Référence électronique}

Jean-Christophe Le Coustumer, « Le contentieux de l'excès de pouvoir des autorisations d'urbanisme

et le droit au recours », Cahiers de la recherche sur les droits fondamentaux [En ligne], 14 | 2016, mis en ligne le 01 octobre 2019, consulté le 14 novembre 2022. URL : http://journals.openedition.org/crdf/

586 ; DOI : https://doi.org/10.4000/crdf.586 


\title{
Le contentieux de l'excès de pouvoir des autorisations d'urbanisme et le droit au recours
}

\author{
Jean-Christophe LE COUSTUMER \\ Professeur de droit public à l'université de Caen Normandie \\ Directeur adjoint du Centre de recherche sur les droits fondamentaux et les évolutions du droit (CRDFED, EA 2132)
}

I. Un office du juge au soutien de la défense de l'acte contesté

A. La limitation des moyens invocables

B. La régularisation des autorisations en cours d'instance

II. Un accès au juge limité

A. Un intérêt à agir redéfini strictement

B. La limitation de l'appel

Le 8 janvier 2016 était signé un décret portant, selon son intitulé, sur les ouvrages de production et de transport d'énergie renouvelable en mer $^{1}$. Mais, comme les commentaires sur ce texte n'ont pas manqué de le noter, ce décret « intéresse en vérité moins les ouvrages de production et de transport d'énergie renouvelable en mer que le contentieux administratif y afférant $»^{2}$. Par ce texte en effet le pouvoir réglementaire définit un régime contentieux spécifique relatif aux actes touchant à la mise en place des différents moyens de production des énergies marines renouvelables (EMR). Ce régime est remarquable en ce qu'il prévoit tout à la fois la détermination d'une juridiction compétente exclusivement en premier et dernier ressort pour juger des contentieux portant sur les décisions nécessaires à la réalisation des projets d'EMR $^{3}$, et des règles procédurales particulières dont l'objectif assumé est de réduire la possibilité des contentieux contre ces décisions ${ }^{4}$. La justification de ces nouveautés procédurales a entièrement tenu à la volonté de venir limiter les « effets» des recours contentieux attendus contre les différents types de projets d'EMR lorsque ceux-ci vont se développer, et notamment dans le domaine des énergies éoliennes. La fiche d'impact du projet de décret énonce en effet très explicitement que:

Dans le cadre juridique actuel ces recours peuvent être paralysants sur de nombreuses années, compte tenu notamment du besoin de sécurité juridique pour lever des fonds nécessaires à la réalisation de ces projets $[\ldots]^{5}$.

Ce souci, affiché clairement par les auteurs du décret, de limiter, tant que faire se peut, les effets des recours contentieux dirigés contre des actes qui permettent la mise en œuvre de projets aux enjeux économiques importants,

1. Décret no 2016-9, 8 janvier 2016, JORF, nº 8, 10 janvier 2016.

2. F. Schneider, «Un régime contentieux spécial pour les projets d'énergies marines renouvelables", L'actualité juridique. Droit administratif, 2016 , p. 485.

3. À savoir la cour administrative d'appel de Nantes.

4. Sont prévues une formalité tenant en une obligation de notification des recours administratifs et contentieux sur le mode de l'article R. 6oo-1 du Code de l'urbanisme déjà existant et une procédure de réclamation parallèle à la procédure contentieuse.

5. Cité par F. Schneider, «Un régime contentieux spécial...», p. 486. 
rejoint celui déjà présenté au soutien de la dernière réforme du contentieux de l'urbanisme par l'ordonnance du 18 juillet $2013^{6}$. Dans un contexte d'inflation législative qui voit se succéder à un rythme de plus en plus rapide des réformes en matière d'urbanisme, l'année 2013 a vu l'adoption d'une ordonnance le 18 juillet et d'un décret le $1^{\text {er }}$ octobre $^{7}$, textes qui ont apporté des évolutions du contentieux de l'urbanisme toutes inspirées par une volonté de mettre en place un nouvel équilibre entre le droit de construire et le droit au recours, nouvel équilibre avant tout fondé sur une meilleure sécurisation des situations juridiques individuelles créées par les autorisations d'occuper les sols qui rendent ces projets possibles. C'est ainsi tout un arsenal visant à permettre qu'il soit jugé plus rapidement et plus efficacement de la légalité des autorisations d'urbanisme qui a été mis en place, et ce dans le but avoué de permettre que les projets de construction soient plus rapidement sécurisés juridiquement ${ }^{8}$ et puissent être mis en œuvre ${ }^{9}$. Et cette sécurisation présentée comme indispensable était envisagée pour venir soutenir la réalisation des projets de construction (notamment de logements) nécessaire au développement de l'activité économique. Dans des termes très semblables à ceux de la fiche d'impact du projet de décret de janvier 2016 sur les EMR précités, l'introduction du rapport remis par le groupe de travail mis en place en 2013 et dirigé par le président Labetoulle estimait partagé le constat:

Que l'épuisement des voies de recours contre une autorisation d'urbanisme puisse n'intervenir qu'au terme de plusieurs années et que cette situation ait actuellement pour effet de retarder d'autant le début de la construction $[\ldots]^{10}$.

Cet enjeu d'une sécurisation de projets aux impacts économiques importants à travers la définition d'un régime contentieux spécifique destiné à limiter la possibilité des recours, tout en accélérant les procédures une fois cellesci engagées, sur fond de définition d'un office du juge adapté, caractérise les préoccupations du législateur et du pouvoir réglementaire depuis maintenant quelques années. Et le contentieux de l'urbanisme est évidemment particulièrement propice à des évolutions s'inscrivant dans cet objectif, au regard de l'objet même des décisions qui sont contestées. Mais si l'objectif poursuivi est louable, il n'en reste pas moins que ce jeu avec les règles du contentieux administratif ne peut évidemment dépasser certaines limites en termes de garantie du droit au recours effectif. Le titre même du rapport précité remis le 25 avril 2013 par le groupe de travail dirigé par le président Labetoulle marque bien cette préoccupation en ce que ce rapport est fort opportunément intitulé Construction et droit au recours: pour un meilleur équilibre. Toute réforme ou, plus modestement, toute modification des règles contentieuses qui vise à limiter les effets des recours, se confronte évidemment à une limite qui est celle du respect du droit au recours juridictionnel effectif. Une chose est, pour le pouvoir politique, de soutenir la réalisation de projets ayant des incidences économiques réelles, une autre est de le faire au détriment de principes fondamentaux touchant à l'exercice d'un droit fondamental tel le droit au recours.

Ce soutien à des intérêts économiques qui pousse à modifier des règles essentielles du droit administratif n'est pas sans poser des questions sur l'évolution du droit administratif lui-même au regard du fait que comme peut l'écrire en effet Xavier Domino,

L'une des tendances à notre sens les plus fortes dans la jurisprudence administrative, c'est que le juge administratif ne se préoccupe plus seulement d'affirmer qu'existe un droit au recours, mais qu'il se soucie de plus en plus de trouver et de forger les voies de recours les plus adaptées aux intérêts spécifiques dont les requérants peuvent vouloir, en fonction des configurations, défendre la préservation face aux agissements de l'administration ${ }^{11}$.

Mais, comme l'écrit également Frédéric Rolin, sur un ton un peu plus provocateur:

Ce mouvement de la jurisprudence, qui vise à renforcer la protection des intérêts économiques en leur réservant un traitement juridique propre plus favorable constitue, de notre point de vue, une rupture du "pacte républicain", non pas au sens que lui donne le débat public contemporain, mais à celui que lui donnait Romieu dans ses conclusions sur l'arrêt Martin [... $]^{12}$.

En tout état de cause, force est de constater que le contentieux de l'urbanisme, et encore plus particulièrement le contentieux de l'excès de pouvoir des autorisations d'occuper les sols, poussé par cette volonté de protection des intérêts économiques attachés la réalisation de projets de construction, a atteint aujourd'hui un degré d'autonomie notable par rapport au contentieux administratif général en ce que de nombreuses règles touchant tant à l'office du

6. Ordonnance $\mathrm{n}^{\circ} 2013-638$ du 18 juillet 2013, JORF, $\mathrm{n}^{\circ} 166,19$ juillet 2013, p. 12070.

7. Décret $n^{\circ} 2013-879$ du $1^{\text {er }}$ octobre 2013 relatif au contentieux de l'urbanisme.

8. C'est-à-dire purgés de tout recours, gracieux ou contentieux.

9. Voir J.-C. Le Coustumer, «Faire face efficacement au contentieux des autorisations d'urbanisme», L'actualité juridique. Collectivités territoriales, mai 2015, p. 257-259.

10. Construction et droit au recours: pour un meilleur équilibre, rapport remis le 25 avril 2013 à $\mathrm{M}^{\mathrm{me}}$ Cécile Duflot, ministre de l’Égalité des territoires et du Logement, en ligne: www.logement.gouv.fr/IMG/pdf/Rapport_Labetoulle.pdf, p. 1.

11. X. Domino, «Droit au recours et équité du procès devant la justice administrative aujourd'hui», in Les nouveaux cahiers du Conseil constitutionnel, $\mathrm{n}^{\mathrm{o}}$ 44, juin 2014, en ligne: http://www.conseil-constitutionnel.fr/conseil-constitutionnel/francais/nouveaux-cahiers-du-conseil/cahier-n-44/ droit-au-recours-et-equite-du-proces-devant-la-justice-administrative-aujourd-hui.1416o1.html.

12. L'auteur poursuit en estimant que «ce pacte républicain qui faisait partie de la justice administrative qui accompagnait le mouvement d'adoption des grandes lois de libertés publiques sous la III République, un organe démocratique chargé du contrôle de l'administration, ouvrant pour cette raison largement le prétoire aux citoyens conçus comme des initiateurs, voire les agents de ce contrôle qui visait à "censurer l'illégalité, éclairer l'opinion publique, et [...] prévenir le retour des pratiques condamnées" » (F. Rolin, "Le droit administratif est-il au service du Grand Capital ? ", L'actualité juridique. Droit administratif, 2016, p. 926) 
juge qu'à l'accès même au prétoire ont été progressivement mises en place au rythme de textes successifs. Si le droit au recours, au sens des dispositions internationales qui le reconnaissent ${ }^{13}$, et au sens des dispositions nationales pertinentes, ne semble pas directement atteint par une disposition ou une jurisprudence particulière, en revanche une vraie question se pose quant au cumul de règles procédurales qui, évaluées dans leur ensemble, dressent un tableau qui amène aujourd'hui à douter très sérieusement de l'effet utile d'un recours pour excès de pouvoir dirigé contre une autorisation d'urbanisme.

Le contentieux de l'excès de pouvoir des autorisations d'urbanisme présente en effet des particularités multiples tenant à la fois à l'office du juge administratif, qui apparaît adapté à l'objectif de protection des autorisations (I), et aux conditions mêmes d'accès au juge, qui font l'objet de conditions limitatives notables (II).

\section{Un office du juge au soutien de la défense de l'acte contesté}

L'office du juge administratif en matière de contentieux de l'urbanisme, et particulièrement pour les autorisations d'urbanisme, connaît des spécificités notables qui dérogent à l'office «classique» des juridictions administratives dans le cadre de l'excès de pouvoir. Et certaines de ces spécificités ont été mises en place pour limiter l'étendue du débat judiciaire ainsi que les effets des décisions des juges, et ce au soutien des intérêts du bénéficiaire de l'autorisation d'urbanisme contestée.

\section{A. La limitation des moyens invocables}

Le pouvoir réglementaire a tout d'abord voulu permettre que les débats devant le juge de l'excès de pouvoir soient contraints par des règles qui limitent l'étendue des arguments soulevés par le requérant. Deux séries de mesures touchant, de façon générale, au contentieux des permis de construire ou d'aménager, ou de façon particulière à l'urbanisme commercial, ont ainsi été adoptées.

En premier lieu, en ce qui concerne le contentieux des autorisations, le décret du $1{ }^{\text {er }}$ octobre 2013 déjà mentionné a ainsi prévu qu'à la demande motivée d'une partie le juge pourra fixer une date à partir de laquelle aucun nouveau moyen ne pourra être produit devant lui ${ }^{14}$. Cette possibilité ouverte au juge, que la collectivité ou le pétitionnaire doivent solliciter expressément, permet d'empêcher que le requérant prolonge volontairement les échanges contradictoires par la production de nouveaux moyens, et ce jusqu'à une clôture de l'instruction qui peut même avoir à être reportée pour permettre aux défendeurs de pouvoir répondre à ces nouveaux moyens successivement soulevés. Avant l'adoption de cette règle, le procès pouvait être ainsi très facilement prolongé, la commune et le pétitionnaire devant pouvoir répondre aux nouveaux moyens soulevés (volontairement) tardivement ${ }^{15}$. Cette modification permet donc incontestablement, si les communes et les bénéficiaires de l'autorisation utilisent cette possibilité en n'oubliant pas de motiver (sérieusement) leur demande comme l'exige le texte du décret, de rationaliser le procès administratif en matière d'urbanisme en mettant un terme à cette pratique de guérilla contentieuse qui prolonge les instances parfois de façon heurtée et donc assez incertaine (clôture, réouverture d'instruction ${ }^{16}$, report d'audience, etc.). Mais si rationalisation il y a effectivement, il n'en reste pas moins qu'il s'agit là d'une limitation certaine à la liberté des débats et des échanges contradictoires devant le juge administratif.

Le législateur est, en second lieu, allé encore un peu plus loin, et ce dans le domaine de l'urbanisme commercial, en protégeant de façon explicite les permis de construire tenant lieu d'autorisation d'exploitation commerciale. Cette association des procédures de permis de construire et d'autorisation d'exploitation commerciale fut une grande nouveauté de la loi no 2014-626 du 18 juin 2014 (dite loi Pinel). L'article L. 425-4 du Code de l'urbanisme prévoit suivant cette loi que tout projet d'implantation, d'extension ou de modification d'un projet autorisé, doit faire l'objet d'une demande de permis de construire, permis qui tiendra lieu d'autorisation d'exploitation commerciale. L'article L. 60o1-4 du Code de l'urbanisme, créé par cette loi Pinel, prévoit en effet que les recours dirigés contre de telles autorisations ne pourront porter que sur l'autorisation commerciale lorsque le recours est engagé par les personnes visées dans la procédure de délivrance de l'autorisation commerciale, et uniquement sur l'autorisation de construire lorsque le recours est engagé par un requérant autre, à savoir ceux visés par l'article L. 600-1-2 pour la définition de l'intérêt à agir dans le cadre de tels recours ${ }^{17}$.

13. Ce recours est reconnu notamment par la Déclaration universelle des droits de l'homme en son article 8 et par la Convention européenne de sauvegarde des droits de l'homme et des libertés fondamentales en son article 13.

14. La règle a été introduite par l'article R. 600-4 du Code de l'urbanisme.

15. La jurisprudence administrative fait en effet preuve d'une certaine souplesse sur cette question, seule étant exigée la production d'un moyen de légalité externe et d'un moyen de légalité interne avant la fin du délai de recours contentieux (CE, 20 février 1953, Société Intercopie, Recueil Lebon, p. 88).

16. Ou, pire encore, utilisation des dispositions de l'article R. 611-11-1 du Code de justice administrative qui permettent d'informer que la clôture est susceptible d'intervenir à tout moment sans autre mise en demeure à partir d'une date fixée par la juridiction.

17. Art. L. 600-1-4 du Code de l'urbanisme: «Lorsqu'il est saisi par une personne mentionnée à l'article L. 752-17 du code de commerce d'un recours pour excès de pouvoir dirigé contre le permis de construire mentionné à l'article L. 425-4 du présent code, le juge administratif ne peut être saisi de conclusions tendant à l'annulation de ce permis qu'en tant qu'il tient lieu d'autorisation d'exploitation commerciale. Les moyens relatifs à la régularité de ce permis en tant qu'il vaut autorisation de construire sont irrecevables à l'appui de telles conclusions. Lorsqu'il est saisi par une personne mentionnée à l'article L. 600-1-2 d'un recours pour excès de pouvoir dirigé contre le permis de construire mentionné à l'article L. 425-4, le juge administratif ne peut être saisi de conclusions tendant à l'annulation de ce permis qu'en tant qu'il vaut autorisation de construire. Les moyens relatifs à la régularité de ce permis en tant qu'il tient lieu d'autorisation d'exploitation commerciale sont irrecevables à l'appui de telles conclusions ». 
Le législateur a ainsi rendu impossible la contestation globale de l'autorisation d'urbanisme et de l'autorisation d'exploitation, dans le but de sécuriser l'une ou l'autre selon le recours qui est engagé. Cette disposition permet ainsi indirectement de distinguer l'appréciation des deux intérêts différents à agir en jeu, celui attaché à la procédure d'autorisation d'exploitation et celui attaché à la contestation de l'autorisation d'urbanisme elle-même, amis au prix d'une restriction importante des moyens qui peuvent être soulevés par les requérants.

\section{B. La régularisation des autorisations en cours d'instance}

Au-delà de cette volonté d'améliorer le déroulement même de la procédure, l’ordonnance de juillet 2013 a voulu également permettre que le procès engagé ait une fonction utile, ce en saisissant l'occasion du contentieux mis en œuvre pour tenter de purger l'autorisation attaquée des vices dont elle serait susceptible d'être entachée.

Les nouvelles dispositions de l'article L. 600-5-1 du Code de l'urbanisme ${ }^{18}$, applicables aux instances en cours au moment de l'entrée en vigueur de l'ordonnance ${ }^{19}$, ouvrent en effet la possibilité au juge, de sa propre initiative, après avoir sollicité les observations des parties, de surseoir à statuer en indiquant le vice régularisable et le délai laissé pour cette régularisation ${ }^{20}$. Elles font suite aux dispositions de l'article L. 600-5 du même Code qui permettent que ne soit prononcée qu'une annulation partielle d'une autorisation lorsque le vice cause de l'illégalité est susceptible d'être régularisée ${ }^{21}$. Collectivité et pétitionnaire doivent alors engager une course contre la montre pour que le dossier de permis modificatif soit déposé, instruit et qu'une autorisation modificative soit prise et transmise à la juridiction dans le délai imparti. Comme cela est déjà le cas, car des autorisations modificatives sont possibles qui viennent purger un vice régularisable ${ }^{22}$, la commune (qui engage potentiellement sa responsabilité sur la légalité du permis) doit veiller à anticiper une éventuelle régularisation rendue possible par le juge dès le moyen adverse soulevé, ou à tout le moins dès la demande faite par la juridiction des observations sur l'usage envisagé de cette procédure. Mais ces dispositions, pourtant incontestablement très utiles pour assouplir les conséquences de l'engagement d'un contentieux, posent des questions tenant à l'étendue de leur champ d'application, questions qui se traduisent dans l'identification de ce qui constitue un vice effectivement régularisable susceptible de permettre la délivrance d'une autorisation modificative. Le Conseil d'État a eu à se pencher sur cette question et a récemment précisé encore le champ d'application du permis de construire modificatif en rappelant les mécanismes de régularisation du permis de construire initial. Dans son arrêt SCI Riviera Beauvert $^{23}$, il s'inscrit dans la droite ligne de sa précédente jurisprudence ${ }^{24}$, laquelle rappelait les conditions d'application des articles L. 600-5 et L. 600-5-1 du Code de l'urbanisme, conditions tournant principalement autour de l'appréciation de l'étendue des modifications nécessaires pour la régularisation, modifications qui ne doivent pas aboutir à une remise en cause de la conception générale du projet lui-même ${ }^{25}$.

Le Conseil d'État censure en l'espèce le raisonnement de la cour, en considérant qu'

Après avoir relevé que le permis en litige méconnaissait l'article $\mathrm{UB} 7 \mathrm{du}$ règlement du plan d'occupation des sols de Menton, relatif à l'implantation des constructions par rapport aux limites séparatives, la cour a estimé que la régularisation de ce vice conduirait à un déplacement de l'implantation de la construction projetée d'au moins quatre mètres. En déduisant de ce déplacement que le vice ne pouvait être régularisé par la délivrance d'un permis modificatif, sans rechercher s'il était de nature à remettre en cause la conception générale du projet, la cour a commis une erreur de droit ${ }^{26}$.

La haute juridiction s'attache ainsi directement à la condition tenant à l'évaluation de cette conception générale du projet pour juger de la légalité du permis modificatif, et fait reproche à la cour administrative d'appel de Marseille de s'être bornée à déduire du déplacement du

18. Art. L. 600-5-1 du Code de l'urbanisme: «Le juge administratif qui, saisi de conclusions dirigées contre un permis de construire, de démolir ou d'aménager, estime, après avoir constaté que les autres moyens ne sont pas fondés, qu'un vice entraînant l'illégalité de cet acte est susceptible d'être régularisé par un permis modificatif peut, après avoir invité les parties à présenter leurs observations, surseoir à statuer jusqu'à l'expiration du délai qu'il fixe pour cette régularisation. Si un tel permis modificatif est notifié dans ce délai au juge, celui-ci statue après avoir invité les parties à présenter leurs observations».

19. CE, avis, 18 juin $2014, \mathrm{n}^{\circ} 376760$

20. Ce délai est laissé à la liberté du juge; voir, pour un exemple de délai de trois mois laissé pour régulariser le vice tiré de l'absence des documents exigés par l'article R. 421-2 du Code de l'urbanisme dans un dossier de demande de permis de construire: CAA Nantes, 28 mars 2014 , nº 12 NTooo81. Reste à savoir si tous les vices, notamment ceux tenant à la légalité interne, peuvent être utilement purgés dans le délai qui est laissé par le juge.

21. Art. L. 600-5 du Code de l'urbanisme: «Le juge administratif qui, saisi de conclusions dirigées contre un permis de construire, de démolir ou d'aménager, estime, après avoir constaté que les autres moyens ne sont pas fondés, qu'un vice n'affectant qu'une partie du projet peut être régularisé par un permis modificatif, peut limiter à cette partie la portée de l'annulation qu'il prononce et, le cas échéant, fixer le délai dans lequel le titulaire du permis pourra en demander la régularisation».

22. Cette régularisation pouvant porter sur la légalité externe et sur la compétence du signataire de l'autorisation, voir CE, 27 novembre 2013 , Association Bois-Guillaume Réflexion, $\mathrm{n}^{\circ} 358765$.

23. CE, 30 décembre 2015, SCI Riviera Beauvert, $\mathrm{n}^{\circ} 375276$.

24. CE, $1^{\text {er }}$ octobre 2015, Commune de Toulouse, $\mathrm{n}^{\circ} 374338$.

25. La démarche du juge est en fait pragmatique et progressive, en ce que celui-ci examine, avant de se pencher in fine sur cette appréciation de la remise en cause de la conception générale, si le permis est entaché d'un vice ou d'un ensemble de vices, si alors les autres moyens sont ou non fondés, si alors le vice affecte l'ensemble du projet et enfin si des éléments du dossier permettent de douter de l'achèvement du projet.

26. CE, 30 décembre 2015, SCI Riviera Beauvert, cons. 5 . 
projet l'impossibilité de régularisation par le biais d'un permis de ce genre. Les juges d'appel avaient effectivement fait l'impasse sur l'étape intermédiaire tenant à examiner si la modification était de nature à remettre en cause la conception générale du projet. Cette dernière notion a été développée par la jurisprudence ${ }^{27}$ et les juges estiment que le permis est modificatif s'il est sans influence sur la conception générale du projet initial, c'est-à-dire si les modifications n'affectent pas la totalité du projet initial. Ainsi, c'est en définissant le champ d'application du permis de construire modificatif que le Conseil d'État précise les conditions d'application des articles L. 600-5 et L. 600-5-1 du Code de l'urbanisme. Mais concrètement, ce champ d'application tel que l'apprécient les juges permet qu'une autorisation modificative intervienne, comme dans l'affaire en cause, de façon assez ouverte au bénéfice du pétitionnaire, permettant ainsi à ce dernier de faire évoluer son autorisation au fil de la procédure et rendant sans objet des moyens du requérant pourtant fondés à l'origine de l'instance.

Il faut noter, et toujours dans le sens de ce même assouplissement, que ce mécanisme a été étendu par la loi ALUR $^{28}$ au contentieux des documents de planification, contentieux particulièrement délicat pour les collectivités territoriales en raison de ses effets ${ }^{29}$. Par l'adoption de l'article L. 600-9 du Code de l'urbanisme ${ }^{30}$, le législateur a autorisé une plus grande souplesse dans ce contentieux spécifique qui permet là encore aux collectivités (ou aux établissements publics de coopération intercommunale quand cela concerne un plan local d'urbanisme intercommunal ou un schéma de cohérence territorial) de sauver leur document de planification d'une annulation totale ou même partielle par le biais de ce dispositif de sursis à statuer ordonné le temps de la régularisation ${ }^{31}$. Cette souplesse législative rejoint celle des juges administratifs eux-mêmes, qui par leur fameuse décision Danthony ${ }^{32}$ ont admis que des actes adoptés après une procédure pourtant entachée d'un vice puissent être sauvés de l'annulation si le vice en question n'est pas susceptible d'avoir eu d'influence sur la décision finale et n'a pas pu léser le requérant dans ses droits. Différentes applications récentes de cette jurisprudence dans le domaine de l'urbanisme ont ainsi préservé de l'annulation des documents de planifica- tion $^{33}$ ou des autorisations d'occuper les sols ${ }^{34}$. Sans aller évidemment jusqu'à entériner un laxisme dans le respect des procédures suivies par les collectivités pour l'adoption des actes d'urbanisme, cette position de la jurisprudence permet de sauver de l'annulation des actes qui étaient contestés au regard de violations de la légalité pour le moins ténues et parfois retenues de façon très formelle.

Mais au-delà de cette modulation de l'office du juge ce sont sans doute les particularités tenant à l'accès même au prétoire administratif qui méritent d'être remarquées dans cette évaluation de la confrontation du droit au recours avec les spécificités du contentieux de l’urbanisme.

\section{Un accès au juge limité}

\section{A. Un intérêt à agir redéfini strictement}

L'appréciation de l'intérêt à agir a toujours suscité beaucoup de réflexions et de débats en doctrine. Élément d'appréciation de la recevabilité d'un recours destiné à éviter, même dans le cadre du recours très objectif qu'est le recours pour excès de pouvoir, une trop large ouverture du prétoire administratif, cet intérêt est un enjeu essentiel de l'accès au juge. Dans ses célèbres conclusions sur ce point, Bernard Chenot estimait que:

Celui qui forme un recours pour excès de pouvoir peut fort bien, aujourd'hui, ne pas être directement et personnellement visé par l'acte qu'il attaque, mais il ne suffit pas qu'il montre que cet acte a pour lui des conséquences fâcheuses, il faut encore que ces conséquences placent le requérant dans une catégorie nettement définie d'intéressés ${ }^{35}$.

Et en matière de recours des tiers contre une autorisation d'urbanisme cette question de la définition d'une «catégorie nettement définie d'intéressés» est essentielle car elle se lie évidemment à la sécurité juridique de l'autorisation délivrée. Cet enjeu particulier attaché à la définition de l'intérêt à agir des requérants en matière d'urbanisme s'exprime bien dans le fait que cette question de l'équilibre entre cet intérêt et le droit au recours est le premier point abordé par le groupe de travail emmené par le président Labetoulle. Le rapport notait d'ailleurs sur ce point que le libéralisme de principe qui caractérise l'appréciation de cet

27. Voir notamment CE, 26 juillet 1982, Le Roy, n' 23604

28. Loi n $2014-366$ du 24 mars 2014 pour l'accès au logement et un urbanisme rénové.

29. Voir, sur ce thème, J.-C. Le Coustumer, «Les documents de planification à l'épreuve du contentieux: la recherche d'un juste équilibre entre sécurité juridique et droit au recours ", in Quel droit pour un urbanisme durable?, V. Le Grand (dir.), Paris, Mare \& Martin, 2014, p. 43-66.

30. «Si le juge administratif, saisi de conclusions dirigées contre un schéma de cohérence territoriale, un plan local d'urbanisme ou une carte communale, estime, après avoir constaté que les autres moyens ne sont pas fondés, qu'une illégalité entachant l'élaboration ou la révision de cet acte est susceptible d'être régularisée, il peut, après avoir invité les parties à présenter leurs observations, surseoir à statuer jusqu'à l'expiration du délai qu'il fixe pour cette régularisation et pendant lequel le document d'urbanisme reste applicable [...]».

31. Le dispositif de l'article L. 6oo-9 CU est complexe en ce que cette régularisation n'est possible, en cas de vice de forme ou de procédure, qu'en cas de vice susceptible d'être purgé par une procédure de modification visée par le Code et uniquement si le vice est apparu dans une formalité intervenue après le débat sur les orientations du PADD (projet d'aménagement et de développement durable).

32. CE, 23 décembre 2011, Danthony, $\mathrm{n}^{\circ} 335033$.

33. CE, 17 juillet 2013, Commune d'Arcachon, $\mathrm{n}^{\circ} 350380$.

34. CE, 4 février 2013, Commune de Saint-Lanne, $\mathrm{n}^{\circ} 335589$. Pour une application à la procédure du retrait de permis, voir CE, 24 mars 2014, Commune de Luc-en-Provence, $\mathrm{n}^{\circ} 356142$.

35. Conclusions sur CE, 10 février 1950, Sieur Gicquel, Recueil Lebon, p. 99. 
intérêt à agir «a fait l'objet [...] de nombreux commentaires critiques de la part des constructeurs essentiellement $»^{36}$. Il est en effet clair que la «facilité» avec laquelle un recours contre une autorisation d'urbanisme peut-être engagé, notamment par la mise en œuvre d'un simple recours gracieux, déconcerte toujours le bénéficiaire de l'autorisation qui voit dans cet engagement d'une contestation sans examen de recevabilité ou de crédibilité préalable une trop grande insécurité juridique.

Cette critique récurrente a été entendue par le législateur puisque la modification du Code de l'urbanisme suite au rapport Labetoulle ayant suscité peut-être le plus d'attention et de commentaires est celle amenée par l'ordonnance du 18 juillet 2013 qui porte sur la redéfinition des contours de l'intérêt pour agir des requérants contre les autorisations d'urbanisme ${ }^{37}$. Pour la première fois, le Code de l'urbanisme accueille en effet un article qui prévoit que, pour être recevable, un recours dirigé contre une autorisation d'urbanisme ${ }^{38}$ doit justifier du fait que:

[...] la construction, l'aménagement ou les travaux sont de nature à affecter directement les conditions d'occupation, d'utilisation ou de jouissance du bien que [le requérant] détient ou occupe régulièrement ou pour lequel [il] bénéficie d'une promesse de vente, de bail, ou d'un contrat préliminaire mentionné à l'article L. 261-15 du code de la construction et de l'habitation ${ }^{39}$.

L'intérêt donnant qualité pour agir était évidemment déjà précisément défini par la jurisprudence administrative avant l'adoption de ce texte. La recevabilité d'un recours contre une autorisation d'urbanisme était soumise à l'évaluation du caractère à la fois approprié et direct de l'intérêt invoqué par le requérant. Cet intérêt à agir se définissait très largement par le critère dit du «voisinage», composé d'un faisceau d'indices qui faisait varier la recevabilité d'un recours en fonction de la distance avec le projet, l'importance de celui-ci et la nature des constructions envisagées. L'appréciation du caractère «direct» de cet intérêt revenait en quelque sorte déjà à évaluer, mais sans le formuler ainsi, si la situation du voisin contestataire était «affectée» par la construction qui, par l'impact urbanistique qu'elle avait sur sa situation, justifiait qu'il puisse en contester la légalité. Le critère de la visibilité de la construction incarnait particulièrement l'appréciation de cet impact du projet sur la situation du requérant.

Mais les dispositions du nouvel article L. 600-1-2 du Code de l'urbanisme sont indéniablement et intentionnellement plus précises sur le point de l'établissement, par celui qui conteste une autorisation, de ce qu'il est effectivement affecté de façon directe dans les conditions d'occupation, d'utilisation ou de jouissance de son bien (qu'il possède ou qu'il occupe régulièrement). La question s'est immédiatement posée, à la lecture de ces nouvelles dispositions, du rapprochement de l'appréciation de cet intérêt à agir avec une appréciation de l'existence d'un préjudice subi par le tiers requérant. Ces termes d' «affectation directe» amenaient en effet à se demander si l'intérêt à agir n'allait pas être soumis à l'établissement de l'existence d'un dommage, même conçu largement, que le projet de construction ferait subir à la situation juridique du requérant. Il faut noter d'ailleurs que, comme ne manquait d'ailleurs pas assez significativement de le rappeler le rapport du groupe de travail Labetoulle, ce lien entre la recevabilité d'un recours et l'appréciation d'une situation préjudiciable pour le requérant n'est pas inconnu en ce que l'article R. 514-3-1 du Code de l'environnement prévoit qu'un certain nombre de décisions ${ }^{40}$

[...] peuvent être déférées à la juridiction administrative: par les tiers, personnes physiques ou morales, les communes intéressées ou leurs groupements, en raison des inconvénients ou des dangers que le fonctionnement de l'installation présente pour les intérêts mentionnés aux articles L. 211-1 et L. 511-1 [... $]^{41}$. (nous soulignons)

Et le Conseil d'État a eu l'occasion d'accepter de lier clairement l'intérêt des requérants et l'existence d' «inconvénients » et de "dangers » attachés pour les requérants à la présence de l'installation concernée ${ }^{42}$. Si en matière environnementale le lien entre la recevabilité d'un recours et une atteinte à un intérêt environnemental, au sens large du terme, se comprend aisément, ce même lien entre une situation préjudiciable trouvant sa source dans une construction, quelle qu'elle soit, et un intérêt à agir en matière d'urbanisme est plus délicat.

Les dispositions de l'article L. 600-1-2 du Code de l'urbanisme n'étant applicables qu'aux recours engagés après l'entrée en vigueur de l'ordonnance du 18 juillet 2013, soit le 19 août 2013, il a fallu attendre quelques mois pour que le conseil d'État puisse être saisi d'une affaire lui permettant de se prononcer sur les modalités de mise en œuvre de ce nouvel intérêt à agir et par là sur l'évolution de celui-ci. Et c'est dans un arrêt du 10 juin 2015 que la haute juridiction est venue préciser le modus operandi de l'appréciation de cet intérêt permettant l'accès au prétoire administratif. Les juges commencent ainsi par préciser qu'il appartient à tout requérant

36. Construction et droit au recours..., p. 7 .

37. À l'exclusion des déclarations préalables exclues de cette réforme.

38. Seuls sont visés par cette redéfinition de l'intérêt à agir les permis de construire, d'aménager ou de démolir, mais pas les déclarations préalables.

39. Art. L. 600-1-2 du Code de l'urbanisme. Cette définition de l'intérêt à agir pèse sur les requérants qui, comme le précise le texte ne sont ni «l'État, les collectivités territoriales ou leurs groupements ou une association ».

40. Les décisions mentionnées au I de l'article L. 514-6 et aux articles L. 211-6, L. 214-10 et L. 216-2.

41. À savoir «des dangers ou des inconvénients soit pour la commodité du voisinage, soit pour la santé, la sécurité, la salubrité publiques, soit pour l'agriculture, soit pour la protection de la nature, de l'environnement et des paysages, soit pour l'utilisation rationnelle de l'énergie, soit pour la conservation des sites et des monuments ainsi que des éléments du patrimoine archéologique» (art. 511-1 du Code de l'environnement).

42. CE, 13 juillet 2012, Société Moulins Soufflet, nº 339592. 
[...] de préciser l'atteinte qu'il invoque pour justifier d'un intérêt lui donnant qualité pour agir, en faisant état de tous éléments suffisamment précis et étayés de nature à établir que cette atteinte est susceptible d'affecter directement les conditions d'occupation, d'utilisation ou de jouissance de son bien $[\ldots]^{43}$.

Ils font ainsi peser sur le requérant une charge initiale de la preuve assez poussée même dans les nouveaux termes de l'article L. 600-1-2 du Code de l'urbanisme. Il s'agit en effet pour celui qui veut voir son intérêt reconnu de produire des éléments étayés et précis qui prouvent l'atteinte invoquée. Mais, cette preuve une fois apportée, les juges précisent qu’il

[...] appartient au défendeur, s'il entend contester l'intérêt à agir du requérant, d'apporter tous éléments de nature à établir que les atteintes alléguées sont dépourvues de réalité $[\ldots]^{44}$.

C'est donc un échange d'arguments étayés sur les éléments apportés au soutien de la démonstration de l'affectation directe du requérant qui s'organise devant les juges qui ont pour charge alors de trancher en évaluant la réalité de cette affectation. Et cette dernière, qui doit être directe, ce qui renforce la restriction envisagée à la détermination de cet intérêt à agir, peut se caractériser par des éléments qui présentent, pour reprendre les termes de Bernard Chenot, des "conséquences fâcheuses " pour le requérant, telles une perte de vue, d'ensoleillement, une modification substantielle des conditions de son environnement à travers l'augmentation sensible de la circulation, des nuisances sonores, etc. Et ces conséquences fâcheuses se rapprochent évidemment de façon claire de l'établissement d'un préjudice direct subi par le requérant dans ces conditions particulières d'occupation de son bien. Il s'agit là donc, d'une certaine façon, d'une qualification d'un intérêt à agir qui s'établit sur le mode de la démonstration d'un dommage anormal attaché à la condition spécifique du requérant, c'est-à-dire sur le mode de la démonstration d'une responsabilité sans faute ou d'un trouble anormal du voisinage. Même si le Conseil d'État a semblé redonner récemment au voisin immédiat du projet contesté une place relativement privilégiée dans l'établissement de cet intérêt ${ }^{45}$, le critère plus objectif du voisinage a donc bien été remplacé par un critère plus restrictif qui rapproche l'établissement de l'intérêt à agir de la démonstration d'une atteinte à une situation juridique envisagée restrictivement.

Mais la modification sans doute la plus délicate issue de cette réforme de l'intérêt à agir, et qui a suscité le plus d'attention de la part des autorités qui délivrent les autorisations, est celle qui touche à la date à laquelle cet intérêt doit dorénavant être apprécié. Suite là encore à une proposition du groupe de travail Labetoulle, un nouvel article L. 600-1-3 du Code de l'urbanisme vient prévoir en effet, que, sauf «circonstances particulières » ${ }^{46}$, "l'intérêt pour agir contre un permis de construire, de démolir ou d'aménager s'apprécie à la date d'affichage en mairie de la demande du pétitionnaire». Cette disposition revient sur le principe consacré par la jurisprudence selon lequel la date d'appréciation de l'intérêt à agir contre un acte est celle non de l'acte lui-même mais de l'introduction du recours contre cet acte, principe qui permettait en effet de créer un intérêt à agir contre une autorisation d'urbanisme postérieurement à sa délivrance, ce qui était une porte laissée ouverte pour les recours dits «mafieux» destinés uniquement à obtenir un avantage financier en échange d'un désistement. Dorénavant, l'intérêt à agir s'apprécie à la date d'affichage en mairie de la demande de permis, le régime prévu déjà pour les associations ${ }^{47}$ devenant la règle en matière de contentieux des autorisations d'urbanisme. Cette appréciation « rétroactive» de l'intérêt à agir des requérants au regard de la date d'engagement de leur recours contentieux a été jugée conforme à la Constitution par le Conseil constitutionnel saisi d'une question prioritaire de constitutionnalité relative à cet article L. 600-1-1 du Code de l'urbanisme ${ }^{48}$.

La question était délicate et il était défendu devant la juridiction constitutionnelle qu'étaient atteints le droit à un recours juridictionnel effectif, de même, par voie de conséquence, que la liberté d'association. Concernant le droit au recours juridictionnel effectif, les juges du palais Montpensier, estimant que «la protection constitutionnelle du droit au recours protège le droit d'agir pour celui qui existe, non le droit d'exister pour agir ${ }^{49}$, ont jugé que:

[...] la restriction ainsi apportée au droit au recours est limitée aux décisions individuelles relatives à l'occupation ou à l'utilisation des sols; que, par suite, l'article L. 600-1-1 du code de l'urbanisme ne porte pas d'atteinte substantielle au droit des associations d'exercer des recours; qu'il ne porte aucune atteinte au droit au recours de leurs membres $[\ldots]^{50}$.

Si, en effet, les dispositions en question n'interdisent pas qu'une association engage un recours contre une autorisation d'urbanisme, elles ont été clairement dirigées contre la constitution d'association ad hoc destinées à contester un projet particulier. Cette volonté de bloquer le recours d'une association constituée après le dépôt d'une demande d'autorisation a pour raison le fait que

43. CE, 10 juin 2015, Brodelle et Gino, no 386121 , cons. 5 .

44. Ibid.

45. CE, 13 avril 2016, Bartolomei, no 389798

46. "Circonstances particulières" qui restent encore à définir par la jurisprudence.

47. Art. L. 600-1-1 du Code de l'urbanisme, introduit par la loi du 13 juillet 2006.

48. CC, déc. $\mathrm{n}^{\circ}$ 2011-138 QPC du 17 juin 2011, Association Vivraviry.

49. Commentaire de la déc. no 2011-138 QPC du 17 juin 2011, en ligne: http://www.conseil-constitutionnel.fr/conseil-constitutionnel/root/bank/ download/2011138QPCccc_138qpc.pdf, p. 8.

50. CC, déc. $\mathrm{n}^{\circ}$ 2011-138 QPC du 17 juin 2011, Association Vivraviry, cons. 7. 
l'intérêt à agir d'une association s'apprécie différemment de celui des particuliers et que cette appréciation différente pouvait amener à ce qu'une association, dont l'objet et le champ d'intervention géographique étaient suffisamment précisément définis, soit recevable à agir contre une autorisation d'urbanisme alors qu'aucun de ses membres pris individuellement ne l'était. Cela permettait donc que soit contournée, en fonction d'un contentieux spécifique, la jurisprudence sur l'intérêt à agir des particuliers, avec la suspicion sous-jacente de la mise en œuvre d'un recours uniquement destiné à obtenir une compensation financière du porteur du projet en échange d'un désistement ultérieur.

\section{B. La limitation de l'appel}

La réforme du contentieux de l'urbanisme de l'année 2013 a également apporté une évolution procédurale importante, bien que passée relativement inaperçue ou ayant, en tous les cas, fait l'objet de peu de commentaires au regard de son objet.

Au-delà même de la redéfinition stricte des contours de l'intérêt à agir, c'est la mise en œuvre des voies de recours qui a été impactée de façon substantielle par les dispositions du décret du $1^{\mathrm{er}}$ octobre $2013^{51}$. Celui-ci supprime, en effet, pour une durée limitée à cinq $\mathrm{ans}^{52}$, la voie de recours de l'appel pour certains projets portant sur les bâtiments à usage d'habitation ${ }^{53}$ lorsque le projet d'aménagement ou de construction est situé sur une commune qui connaît des difficultés sérieuses d'accès au logement sur l'ensemble de son parc résidentiel. En clair, dans les communes (et leur agglomération) identifiées comme telles (par décret), et non des moindres telles que Paris, Bordeaux, Marseille, Lille, Lyon, etc., l'appel contre les permis de construire ou d'aménager visant ce type de construction est, ni plus ni moins, supprimé. Le rapport remis en avril 2013 par le groupe de travail Labetoulle avait ouvert la voie à une telle évolution en suggérant, non pas de supprimer la voie de recours de l'appel dans certains cas, mais de confier directement compétence aux cours administratives d'appel pour ces mêmes cas. Ce choix d'une compétence de premier et dernier ressort des cours a été fait sans coup férir dans le domaine de l'urbanisme commercial par l'adoption de l'article L. 60o10 du Code de l'urbanisme en matière de contestation des permis de construire valant autorisation d'exploitation commerciale ${ }^{54}$, et ce en fonction d'un objectif explicite de raccourcissement des délais de recours contentieux.

Et pour répondre à ce même impératif tenant à une plus grande efficacité dans le fonctionnement juridictionnel, c'est-à-dire en réalité pour permettre également une plus grande célérité dans le traitement des contentieux contre les autorisations d'urbanisme de façon générale, le groupe de travail avait en effet suggéré cette dispense de premier degré de juridiction à titre expérimental dans un premier temps. Mais cette suggestion avait été entourée d'un luxe de précautions, et il faut le dire, d'un ensemble de réserves qui montraient le peu d'enthousiasme des membres du groupe de travail pour cette réforme. Le rapport rappelait en effet qu'une telle modification de l'ordonnancement juridictionnel et contentieux devait appeler tout à la fois une "concertation approfondie au sein des juridictions administratives $"{ }^{55}$ ainsi qu'une étude d'impact complémentaire pour évaluer les éventuels déséquilibres territoriaux provoqués par une telle mesure. Le même texte rappelait également à juste raison que si l'existence d'un double degré de juridiction n'est pas une garantie constitutionnelle en dehors de la matière pénale, celui-ci constitue « une garantie qui profite, quoiqu'elles en pensent, à l'ensemble des parties au litige $»^{56}$. Le double degré de juridiction a en effet bien été expressément jugé comme ne constituant pas une garantie de nature constitutionnelle par les juges du pavillon Montpensier, par une décision du 12 février $2004^{57}$, principe confirmé ensuite notamment par des décisions QPC du 14 mai $2012^{58}$.

Le dispositif prévu par le pouvoir réglementaire pose néanmoins question quant au respect du principe d'égalité en ce que cette suppression de la voie de l'appel est dépendante de conditions attachées à un régime fiscal

51. Décret $\mathrm{n}^{\mathrm{O}} 2013-879 \mathrm{du} 1^{\mathrm{er}}$ octobre 2013 relatif au contentieux de l'urbanisme.

52. Le dispositif est en effet présenté comme expérimental.

53. Le nouvel article R. 811-1-1 du Code de justice administrative prévoit dorénavant que: «Les tribunaux administratifs statuent en premier et dernier ressort sur les recours contre les permis de construire ou de démolir un bâtiment à usage principal d'habitation ou contre les permis d'aménager un lotissement lorsque le bâtiment ou le lotissement est implanté en tout ou partie sur le territoire d'une des communes mentionnées à l'article 232 du Code général des impôts et son décret d'application».

54. Disposition insérée par la loi Pinel du 18 juin 2014 (précitée).

55. Construction et droit au recours..., p. 25. L'atteinte au double degré de juridiction, finalement retenue à travers la suppression de l'appel pour cette catégorie de projet a fait l'objet d'une vive critique de la part de l'Union syndicale des magistrats administratifs qui dans un communiqué a fait savoir que «L'USMA, qui a émis un vote défavorable sur ce texte lors du CSTA [Conseil supérieur des tribunaux administratifs] du 17 septembre 2013, déplore la mise en place de cette "procédure d'exception", qui accrédite la thèse selon laquelle les difficultés de logement seraient imputables au fonctionnement de la justice administrative. Rien ne permet pourtant de faire un lien entre la crise du logement et l'activité contentieuse. On ne peut que regretter l'empressement avec lequel cette mesure a été adoptée, sans aucune analyse préalable. Le rapport Labetoulle relatif à la réforme du contentieux de l'urbanisme préconisait d'ailleurs que l'adoption, le cas échéant, d'une telle mesure, soit précédée d'une concertation approfondie au sein des juridictions administratives et d'une étude d'impact pour déterminer son application géographique...» (USMA, «Décret du $1^{\text {er }}$ octobre 2013 relatif au contentieux de l'urbanisme: l'USMA déplore la suppression du double degré de juridiction!», 6 octobre 2013, en ligne: http://www. usma.fr/decret-du-1er-octobre-2013-relatif-au-contentieux-de-1-urbanisme-l-usma-deplore-la-suppression-du-double-degre-de-juridiction).

56. Construction et droit au recours..., p. 26.

57. CC, déc. $\mathrm{n}^{\circ}$ 2004-491 DC du 12 février 2004, Loi complétant le statut d'autonomie de la Polynésie française, cons. 4. Le Conseil d'État ne range d'ailleurs pas ce principe dans la catégorie des principes généraux du droit (CE, 17 décembre 2003, Meyet, n²58253).

58. CC, déc. $\mathrm{n}^{\circ}$ 2012-243/244/245/246 QPC du 14 mai 2012, Société Yonne républicaine et autre. 
prévu par l'article 232 du Code général des impôts qui vise les communes où les constructions sont assujetties à une taxe spécifique, celle sur les logements vacants. Et c'est un décret qui fixe quelles sont les communes en question sur la base d'un critère tenant à l'existence d'un « déséquilibre marqué entre l'offre et la demande de logements, entraînant des difficultés sérieuses d'accès au logement sur l'ensemble du parc résidentiel existant ${ }^{59}$. Mais ce texte a donc pour effet de lier, à la discrétion du pouvoir réglementaire, l'absence d'appel possible en matière de contestation d'autorisation d'urbanisme avec un dispositif fiscal de soutien à la construction de logements. Et ce mécanisme a donc pour conséquence indirecte de moduler les voies de recours possibles selon le territoire sur lequel le projet objet de l'autorisation d'urbanisme contesté a été délivré. C'est alors la question de la balance entre cette privation d'une voie de recours au fond contre une décision juridictionnelle et l'intérêt général attaché à ce dispositif de soutien financier qui se pose au regard du principe d'égalité, et plus particulièrement du principe d'égalité devant la justice.

On le sait, de façon générale, le Conseil constitutionnel, depuis une décision du 7 janvier 1988, juge que:

[...] le principe d'égalité ne s'oppose ni à ce que le législateur règle de façon différente des situations différentes ni à ce qu'il déroge à l'égalité pour des raisons d'intérêt général pourvu que, dans l'un et l'autre cas, la différence de traitement qui en résulte soit en rapport direct avec l'objet de la loi qui l'établit ${ }^{60}$.

Les mêmes juges ont également précisé, relativement à l'appréciation du respect du principe d'égalité appliqué aux droits de la défense, que:

[...] si le législateur peut prévoir des règles de procédure différentes selon les faits, les situations et les personnes auxquelles elles s'appliquent, c'est à la condition que ces différences ne procèdent pas de distinctions injustifiées et que soient assurées aux justiciables des garanties égales, notamment quant au respect du principe des droits de la défense, qui implique en particulier l'existence d'une procédure juste et équitable ${ }^{61}$.

La question est donc de savoir si la distinction faite quant à l'existence ou non d'une procédure d'appel ne constitue pas une distinction injustifiée et si les justiciables bénéficient de garanties égales en matière de contentieux des autorisations d'urbanisme ${ }^{62}$. La justification avancée pour cette suppression de l'appel dans les cas en question tient tout à la fois à la nécessité de permettre la construction de logements dans des zones où celle-ci est nécessaire et à la célérité indispensable des procédures de contestation des autorisations qui risquent de contrarier trop fortement la mise en œuvre desdites constructions. Si l'objectif invoqué est louable sur le principe, on peut s'interroger sur le caractère proportionné de la mesure visant à fermer la voie de l'appel au soutien de celui-ci. En effet, dans la balance entre le respect du droit des justiciables (qu'il s'agisse d'ailleurs des requérants comme des défendeurs) et la poursuite d'un objectif socio-économique de construction de logements, il paraît pour le moins rapide de privilégier le second sur le premier. Et ce d'autant plus que la différence de traitement est très géographiquement déterminée. Les territoires visés par cette suppression de l'appel sont en effet très nombreux et concernent les plus grandes agglomérations françaises, touchant ainsi une population assez étendue. Et la règle prévue par le décret du $1^{\mathrm{er}}$ octobre 2013 a pour conséquence que d'une commune à l'autre, même éventuellement dans une même zone urbaine, le même projet pourra ou non faire l'objet d'un appel après une décision de première instance.

Il apparaît ici en quoi cette réglementation est différente de celle présentée en introduction de ces lignes et portant sur la compétence exclusive de premier et dernier ressort attribuée à une cour administrative d'appel relativement aux contestations de décisions relatives aux projets d'EMR ou celle relative aux permis valant autorisation d'exploitation commerciale. L'exclusivité ainsi mise en place, qui s'accompagne d'une suppression de l'appel pour ces contentieux, vise une certaine catégorie d'objets juridiques définie de façon générale, sans référence à un choix du pouvoir réglementaire fondé sur un dispositif de soutien fiscal territorialement établi. L'égalité des requérants potentiels contre les décisions en question est donc préservée, quoique ce choix procédural puisse rester contestable sur le principe.

En conclusion de ces brefs développements, il apparaît nettement que le contentieux de l'excès de pouvoir des autorisations d'urbanisme connaît un grand nombre de spécificités qui font s'interroger sur le degré d'autonomie par lui atteint au regard du contentieux administratif général, mais qui interrogent surtout ce degré d'autonomie en termes de limites aux droits offerts à ceux qui veulent contester la légalité desdits actes. C'est plus à une forme de découragement organisé et intentionnel des velléités contentieuses qu'à une stricte remise en cause du droit au recours effectif à laquelle on assiste. Mais un contentieux dans lequel tout est mis en œuvre procéduralement pour limiter tant l'accès au prétoire que les effets des décisions

59. Décret n 2013-392 du 10 mai 2013 relatif au champ d'application de la taxe annuelle sur les logements vacants instituée par l'article 232 du Code général des impôts.

60. CC, déc. no 87-232 DC du 7 janvier 1988, Loi relative à la mutualisation de la Caisse nationale de crédit agricole, cons. 10.

61. CC, déc. $\mathrm{n}^{\circ}$ 2009-590 DC du 22 octobre 2009, HADOPI, cons. 11.

62. Comme le précise bien F. Mélin-Soucramanien, en cette matière, «Il appert clairement de ce standard jurisprudentiel que l'égalité ne peut être un droit fondamental absolu et inconditionnel, mais au contraire une norme relative et contingente» («Le principe d'égalité dans la jurisprudence du Conseil constitutionnel. Quelles perspectives pour la question prioritaire de constitutionnalité?», Cahiers du Conseil constitutionnel, n 29 , octobre 2010, en ligne: http://www.conseil-constitutionnel.fr/conseil-constitutionnel/francais/nouveaux-cahiers-du-conseil/cahier-n-29/le-principed-egalite-dans-la-jurisprudence-du-conseil-constitutionnel-quelles-perspectives-pour-la-question-prioritaire-de-constitutionnalite.52731.html). 
rendues amène à porter un regard circonspect sur l'étendue réelle du recours exercé. Si l'on ajoute aux constats précédemment présentés que le législateur a rendu assez vaine l'annulation même d'une autorisation d'urbanisme en limitant de façon très sensible les possibilités que soit prononcée une démolition de l'ouvrage irrégulièrement bâti ${ }^{63}$, et a introduit, dans les dispositions mêmes du Code de l'urbanisme, la menace d'une possible action reconventionnelle dirigée contre le requérant si celuici venait à abuser de son droit au recours ${ }^{64}$, alors il est certain que l'utilité d'un recours contre une autorisation d'urbanisme, à défaut de mettre en péril au sens strict le droit au recours juridictionnel effectif, rend peu effectif le recours juridictionnel mis en œuvre. Si le contentieux de l'urbanisme est bien ce «laboratoire du contentieux administratif de demain ${ }^{65}$, il doit rester un laboratoire qui ne prête pas à des expériences qui mettent en péril le juste équilibre entre la protection des intérêts économiques poursuivie et la situation des requérants légitimes à agir contre une autorisation d'urbanisme.

63. Art. L. 480-13 du Code de l'urbanisme tel que modifié après adoption de la loi dite Macron: loi no 2015-990 du 6 août 2015 pour la croissance, l'activité et l'égalité des chances économiques.

64. Art. L. 600-7 du Code de l'urbanisme qui prévoit que: «Lorsque le droit de former un recours pour excès de pouvoir contre un permis de construire, de démolir ou d'aménager est mis en œuvre dans des conditions qui excèdent la défense des intérêts légitimes du requérant et qui causent un préjudice excessif au bénéficiaire du permis, celui-ci peut demander, par un mémoire distinct, au juge administratif saisi du recours de condamner l'auteur de celui-ci à lui allouer des dommages et intérêts. La demande peut être présentée pour la première fois en appel ».

65. L. Dutheillet de Lamothe, G. Odinet, "Contentieux de l'urbanisme: poursuite de la construction ", L'actualité juridique. Droit administratif, 2016, p. 950. 